Revue de philosophie et de sciences humaines

e-Portique | 2005

\title{
La liberté comme idée, et comme réalité
}

Deux sens nécessaires pour la liberté

Jean-Michel Charrue

\section{OpenEdition}

1 Journals

Édition électronique

URL : https://journals.openedition.org/leportique/654

DOI : 10.4000/leportique.654

ISSN : $1777-5280$

Éditeur

Association "Les Amis du Portique"

Référence électronique

Jean-Michel Charrue, «La liberté comme idée, et comme réalité », Le Portique [En ligne], e-Portique, mis en ligne le 16 juin 2005, consulté le 10 septembre 2022. URL : http://journals.openedition.org/ leportique/654 ; DOI : https://doi.org/10.4000/leportique.654

Ce document a été généré automatiquement le 10 septembre 2022.

Tous droits réservés 


\section{La liberté comme idée, et comme réalité}

Deux sens nécessaires pour la liberté

Jean-Michel Charrue

1 Ces quelques lignes avaient été l'objet d'un cours précédé d'une enquête sur les définitions possibles de la liberté; on ne pouvait pas inaugurer à l'avance des réponses, mais l'usage nous avait appris qu'elles traduisent des mentalités, et que celles-ci ne sont pas ce qu'elles seraient devant d'autres publics d'autres pays : "nos concepts, disions-nous, sont historico politiques ", marqués par l'histoire : la liberté avait-elle le même sens pour un citoyen soviétique, ou pour un africain ? C'est l'histoire d'un peuple qui a façonné ses mentalités. Les réponses nous paraissent pouvoir être réparties à l'intérieur de notre plan, aux différents niveaux de celui-ci.

2 La sémantique (discours chargé de sens de sémainein, signifier) des discours politiques sur la liberté recèle une intense contradiction : une pièce de 1 franc, ou maintenant un euro porte la devise: liberté , égalité fraternité, qui affirme dans l'article 4 de la proclamation des droits de l'homme et du citoyen du 4 Août 1789 : « la liberté est le pouvoir de faire tout ce qui ne nuit pas à autrui....les bornes doivent en être fixées par la loi »; le discours de Danton ou Robespierre, avaient porté au firmament la liberté; d'autre part, chacun sait qu'il y a eu 1793, et que la terreur a guillotiné au nom de cette liberté.

On retrouverait chez Marcuse la même contradiction dans l'Homme unidimensionnel où le porte parole de la contestation dans les universités américaines marque la différence entre des formules telles que "la défense du monde libre" et les bombes qui s'abattaient au Viêt-Nam; tous les pires dictateurs ont parlé de liberté, on n'insistera pas sur la formule qui figurait dans les camps nazis : «le travail rend libre ». Revenons à notre exemple de 1789 : selon nous la contradiction ne peut se résoudre qu'à l'intérieur d'une dualité :

4 (1) la liberté comme mot, concept, idée

5

(2) la liberté comme réalité 
7 en même temps qu'en montrant que nos conceptions en France de la liberté, des réponses de nos élèves sont marquées par 1789, et par l'histoire qui est en nous.

I La liberté comme mot, concept et idée

8 L'étude des origines renseigne la plupart du temps sur un sens porté par une notion: on le trouve dans les Ennéades de Plotin, où l'origine a une grande place, chez Nietzsche, ou encore Bergson qui dans les Deux sources de la morale et de la religion, le note à propos de la justice.

La théorie du péché originel

Or l'origine de la liberté prend sa source dans un passage précis de la Bible:

10 bon

11 Dieu a créé Adam

12 libre

13 Seule l'invention du mot et du concept de liberté rend compte du péché originel, car tout autre explication fait se rejeter la faute sur Dieu, particulièrement celle du mal moral. Au XVIII è siècle, Voltaire avec le tremblement de terre de Lisbonne, ou de tous temps les hommes: si Dieu existe : laisserait-il faire le mal? La liberté ainsi inventée expurge la faute de Dieu à l'intérieur d'une contradiction entre bonté et liberté chez l'homme : en même temps elle commence à expliquer la tournure au départ idéaliste de la liberté, puisque celle-ci apparaît dans un discours religieux sur l'homme en présence du péché originel.

14 La théorie trouve quelques applications chez deux philosophes : Leibniz et J. P.Sartre.

15 Le premier hérite de la théorie du christianisme dont il se fait l'écho, dans les Essais de Théodicée : "comment l'âme a pu être infectée du péché originel...sans qu'il y ait eu l'injustice en Dieu », et que : "Dieu a fait l'homme à son image, mais il l'a fait libre... ", cette liberté est « intelligence, qui en est comme l'âme... (ce qui fait que) la substance libre se détermine par elle-même $»^{1}$. Seulement l'harmonie universelle qui accordait toutes les libertés entre elles était établie par Dieu. C'est un passage du Discours de Métaphysique qui note: "nous soutenons que tout ce qui doit arriver à quelque personne est déjà compris virtuellement, dans sa nature ou sa notion comme les propriétés le sont dans la définition du cercle ». Et il prendra l'exemple de J. César, dont le fait «qu'il deviendra dictateur et maître de la République est déjà compris virtuellement dans sa notion». Dans ses gènes? Il est vrai que Leibniz fera la distinction là encore entre ce qui arrive nécessairement et ce qui n'arrive que par hypothèse, ou accidentellement, pour dire qu'il maintient la liberté.

16 On sait que Sartre s'est accaparé de l'analyse de Leibniz, de façon magistrale pour fonder l'existentialisme de L'Etre et le Néant, déclarant « Un autre geste d'Adam n'eut été possible que par et pour un autre Adam, et l'existence d'un autre Adam impliquait celle d'un autre monde...nous reconnaissons avec Leibniz que le geste d'Adam engage la personne d'Adam entière et qu'un autre geste se fût compris à la lueur et dans les cadres d'une autre personnalité d'Adam. Mais Leibniz retombe dans un nécessitarisme opposé à l'idée de liberté lorsqu'il place la formule de l'existence d'Adam comme une prémisse qui amènera l'acte d'Adam.......il en résulte que l'acte est rigoureusement nécessité par l'essence même d'Adam ". ${ }^{2}$

17 C'est alors que Sartre saute le pas et dit, « Adam a choisi de prendre la pomme, mais n'a pas choisi d'être Adam. Pour nous, en effet, c'est au niveau du choix d'Adam par lui- 
même, c'est à dire de la détermination de l'essence par l'existence que se place le problème de la liberté.... l'existence précède l'essence » et c'est la condition même de la liberté. L'existentialisme est né de cette formule. En toute rigueur elle apparaît vraie: Adam est-il pécheur avant d'avoir péché ? Non, donc Sartre a raison. Mais à bien y réfléchir l'objection leibnizienne a quand même quelque poids, lorsqu'on prend un exemple moderne des violeurs d'enfants ; n'y a-t-il une nature devenue mauvaise qui les poussera à recommencer? Tous les tribunaux savent cela. Les problèmes essentiels sont posés : une liberté qui est le surgissement de l'existence, et le péché originel s'adressant à un acte initial, instaurateur, et à l'autre bout une nature humaine qui serait comme préformée, déterminée. Le fait que ce soit un débat théologique qui ait suscité l'idée de liberté contribue, bien sûr, à lui donner son tour idéaliste.

La théorie du libre-arbitre

18 Celle-ci va contribuer extraordinairement à renforcer cet aspect. Parmi toutes les définitions possibles de la liberté, la plus caractéristique, comme la plus facile à retenir nous parait être celle de Descartes, dans la IV è Méditation : « la liberté, c'est le pouvoir de faire ou de ne faire pas ». Le langage cartésien se traduit par ne faire pas c'est à dire une inversion de la formule telle qu'elle est employée de nos jours : ne pas faire, mais elle traduit dans son raccourci peut-être le mieux la liberté.

19 Descartes se rapproche ainsi de la théorie du libre arbitre qu'il n'a pas inventée, mais dont il est redevable. On sait que Saint Augustin avait dans le Traité du libre arbitre, fait la différence entre une liberté qui ne pouvait être que bonne, vouloir le bien, et un libre arbitre qui pouvait aussi bien faire le mal. Or la formule de Descartes maximalise de nouveau la liberté, qui dans la IV è Méditation peut n'être qu'infinie à l'instar de celle de Dieu, ainsi cette liberté équivalente strictement au vouloir ( voluntas sive libertas, la volonté ou bien la liberté), et elle peut conduire à refuser son assentiment même au cogito, je pense donc je suis, dont je peux me détourner comme je peux me détourner de toute autre considération, lui refuser mon attention et mon intérêt : je ne considère que ce que je veux, comme je ne fais que ce que je veux bien faire par cette liberté totale qui est aussi libre arbitre. L'exemple est-il né de la vision de l'âne de Buridan au XIV è siècle qui, au milieu d'un seau d'eau et d'un seau d'avoine, également affamé et assoiffé, ne se déterminait jamais, peut-être parce que c'était le plus bas degré de cette liberté, la liberté d'indifférence? Mais en outre, parce que la liberté devient un pouvoir de choix, qui sera plus propre à l' homme..

20 Il reste que la liberté était ainsi définie était totale. Est-elle purement théorique ? Un manuel de philosophie notait: «Lalande définissait la liberté ainsi entendue comme une sorte de passage à la limite: on se représente l'action comme successivement affranchie de toutes les causes », puis ajoutait: " la liberté ainsi entendue est le pouvoir d'agir indépendamment non seulement des contraintes extérieures, mais de toute détermination intérieure ; tel est le libre arbitre des métaphysiciens $»^{3}$.

21 Les deux formules traduisent les deux déterminismes les plus forts qui sont devenus ceux de Sigmund Freud, déterminisme intérieur, par les pulsions, la libido, ou encore les instincts agressifs et érotiques dont parlera Malaise dans la Civilisation, puis le déterminisme marxiste, celui du milieu social et de l'éducation.

Liberté ou déterminisme? telle est la grande question, que rencontre notre débat: l'homme est-il un être libre ou déterminé ? Vision de l'homme qu'elle sous-tend. Il est vrai que la théorie du libre arbitre était parfaitement idéaliste, l'est toujours mais peuton poser un homme non libre? Nous sommes en train de penser, de faire de la 
philosophie, que vaut celle-ci, si c'est le Président de la République, le ministre, une autorité religieuse qui fait ma pensée? Que vaut alors celle-ci? Rien. Seule une philosophie le la liberté est une philosophie digne de ce nom, celle de Descartes, où s'affirme la liberté de l'homme par rapport à Dieu, ou celle de Sartre, d'un homme libre sans Dieu. Il faut postuler la liberté, comme par une sorte de pétition de principe: l'homme est un être libre.

23 Mais cette théorie du libre arbitre était factice en un sens : je suis déterminé. Que j'ai ici et maintenant hic et nunc le pouvoir de dire : « soit je continue mon cours soit je vais à Katmandou ». Qui le croira ? Il y a la réalité, le poids et la pression du monde, les fins de mois. Mais il fallait poser que j'ai le pouvoir de le faire. La liberté était devenue ce pouvoir de choix, il restait théorique, mais il fallait qu'il soit là pour que l'homme soit un homme, c'est à dire cet être libre. La liberté ainsi allait valoir pour les grands choix de l'existence, comme dans le Ou bien ...ou bien de Sören Kierkegaard, existentialiste chrétien du XIX è siècle, « ou bien... j'épouse Cordélia ou bien je ne l'épouse pas ». La liberté dans les choix de vie, du mariage, d'une carrière, celle de l'homme dans son entier.

II La liberté comme réalité

24 Si l'existence la liberté trouve ainsi ses racines dans une origine de l'homme qui font du problème de la liberté un problème métaphysique ( = au-delà de la physique relié à l'âme et à Dieu), ou encore ontologique ( = de l'être, ônn, ontos) c'est à dire de l'être de l'homme, et en effet, chez Descartes la liberté sera ce face à face avec Dieu, puisque dans la tradition chrétienne l'homme a été créé, et comme tel maintiendra une relation privilégiée avec son créateur, donnant à la liberté cette dimension verticale, c'est pourtant sur terre que la liberté va se jouer, et l'on peut dire que son sort ne sera pas aisé.

25 D'abord la liberté existe-t-elle vraiment ? Si la philosophie en a conçu l'existence sur le plan de l'homme celle-ci ne s'impose pas, s'impose même si peu que certains y sont allés de la dénégation: "la liberté ça n'existe pas». C'est à dire que la liberté est tellement insaisissable, qu'on ne peut l'appréhender, la toucher : peut-on saisir la liberté à pleines mains, ou comme l'on peut respirer le dimanche l'air en forêt ? C'est une réalité évanescente ( = qui s'évanouit comme la fumée de cigarettes), et de cette évanescence on a pu parfois douter de son existence.

26 Pour répondre à cela nous avons imaginé la chose suivante: que l'on fasse une pancarte, sur laquelle serait inscrit comme slogan : «la liberté ça n'existe pas », et puis envoyer les manifestants se promener dans la rue de la Santé devant les fenêtres de la prison; inutile de dire ce qui se passera; autrement dit la liberté s'éprouve par la privation de la liberté. On pouvait aller dire à A. Soljenitsyne la même chose, lui qui avait connu le goulag savait bien que cela existait, ceux qui ont connu le Chili de Pinochet ou toutes les dictatures, ou encore les camps ne serait-ce que de prisonniers de la dernière guerre : la liberté s'éprouve par sa négation même dans la privation de liberté.

27 Il est étonnant qu'il faut ainsi en être privé pour savoir qu'elle existe. Serait-ce que nos sociétés en ont rendu la pratique, ou la réalité tellement dérisoire, que celle-ci n'apparaît même plus? Comment apparaît-elle à une conscience? Leibniz parlait d' « un sentiment vif interne de liberté », Descartes disait que « la liberté de notre volonté se connaît sans preuve par la seule expérience que nous en avons». Le sentiment 
intérieur se conjuguera avec une expérience extérieure, et c'est là que la liberté apparaîtra, dans cette sorte de rencontre.

28 C'est vrai qu'on se sent plus ou libre dans un endroit, dans une école, dans une entreprise, dans un pays. Mais cette expérience qui commence en effet à faire passer le problème de la liberté du plan initial qui était le sien de l'homme en général, à un plan politique, où il s'agira des sociétés, autre domaine de la liberté, qui n'est pourtant pas sans lien avec le premier, n'est pourtant pas probante pour tout le monde : Soljenitsyne pouvait ne pas se sentir libre, parce que cela engageait son statut d'écrivain, mais en était-il de même de tous? Tel élève se sentira bien dans un lycée, tel autre non, ou encore un employé dans un bureau. Serait-ce que cette expérience soit purement subjective, ou reliée au statut de la personne? Chacun définit ses libertés en fonction de ses valeurs de ses normes, peut-être aussi de ses pratiques et habitudes de vie; tel intellectuel pourra se sentir libre à Paris de pouvoir aller quand il le veut à la bibliothèque, tel autre pour visiter les musées, là où se déroulera l'espace de leur liberté.

29 La liberté résulte ainsi d'une prise de conscience. Est-ce à dire que plus on prendra conscience plus on sera libre ? Peut-être pas dans l'effectivité même de la liberté, dans sa réalité qui va dépendre de critères plus objectifs, que l'on va voir, mais dans cette part de vécu qui est son effectuation par la personne. Sartre avait fait grincer plus d'un lorsqu'il avait dit: "je ne me suis jamais senti aussi libre que sous l'occupation allemande ». Non pas qu'il puisse être soupçonné de collaboration, lui qui avait créé avec Merleau-Ponty un groupe de résistance rapidement échoué "Socialisme et liberté $\rrbracket^{4}$, mais il expliquera que l'Occupation avait rompre le cercle machinal des habitudes, qu'il fallait soit s'engager, rompre, soit choisir différemment, mais que cela au moins avait fait prendre conscience. La liberté ne serait-elle qu'une conscience de liberté?

30 C'est là que nous pouvons revenir à nos positions initiales des réponses: si dans un public français, on peut constater le plus souvent que prédomine cette première avant toute autre : "être libre, c'est faire ce que l'on veut », c'est par ce côté « anarchisant ", individualiste, à l'opposé d'une position plus légaliste d'une liberté dans la société civile régie et contrebalancée par les lois et les règlements, telle qu'on peut le voir dans d'autres sociétés, comme en Allemagne, c'est disions-nous dans l'introduction, parce que pour nous «être libre, c'est se libérer ». tel est nous semble l'aspect primordial revêtu par le monde moderne dans son appréhension de la liberté. Libération qui s'ajoute à une autre : libération des préjugés, la libération qui résulte de la fin de la dernière guerre, qui porte même ce nom, celle des révolutions de 1830, 1848, de la Commune, libération des mouvements de décolonisation, libération vertigineuse qui atteint toutes les formes de vie, les mœurs, libération de la femme

31 Cette liberté /libération qui, pour nous est celle du monde moderne prenait ses sources dans la révolution. R. Aron pouvait parler dans Histoire et dialectique de la violence, « de la foule en marche qui prenait la Bastille $»^{5}$, de la libération des groupes révolutionnaires, auquel il récusait, contrairement à Sartre un droit à la violence, parce qu'il y avait pour lui la liberté démocratique du suffrage universel. Quelles que soient les positions, néanmoins la liberté est apparue dans ce creuset, violent ou non violent d'une libération de l'homme par lui-même. Et cette libération s'est toujours faite dans un contre ou un anti, pour lequel on peut dire qu'il y a toujours des Bastilles à prendre ou 
des libertés à faire, dans des endroits où elle est plus ou moins bafouée, comme les révolutionnaires à l'encontre de l'ancien régime.

Mais cette liberté-libération, qui pour nous, au moins dans notre société a dominé le monde moderne n'est-elle pas négative? Elle se révèle par une série de refus. Kant avait dans un texte parfois posé au baccalauréat dit, au vu de la révolution, que les serfs d'un propriétaire terrien d'Europe orientale devaient immédiatement être libérés « de ces trois chaînes que sont la famille, l'état et la religion ${ }^{6}{ }^{6}$ Mais l'homme moderne n'en vient-il pas de la sorte à tout nier? Dans le roman de Sartre, L'âge de raison - ce sont mes souvenirs de lecture de terminale- le héros, très peu héros, plutôt même antihéros, Mathieu Delarue, professeur de philosophie, -Sartre ?- veut faire avorter sa maîtresse, à une époque où c'est interdit, parce que comprend on il refuse le mariage aliénation possible ou probable de sa liberté, un peu plus loin, lorsqu'il s'agit d'en chercher les moyens auprès d'un frère avocat ou notaire, critiquera "la famille bourgeoise » ou la famille tout court, plus avant lorsqu'un de ses amis militant communiste le pousse à s'engager à aller faire la guerre d'Espagne refusera cette autre entrave à sa liberté; à la fin du roman on voit Mathieu descendre l'escalier, se regarder : ce qui m'inspirait la réflexion: "être libre, c'est n'être rien »! On peut refuser la famille, la patrie, la religion comme autant d'entraves à la liberté, que reste-t-il ?

33 Et ce sera peut-être le drame du monde moderne, ou à force de retirer les non libertés on arrive à un vide à une sorte de néant. Seul $\mathrm{A}$. Camus, nous semble-t-il, $\mathrm{y}$ a pallié quelque peu lorsqu'il a dit dans le Discours de Suède: non, nous ne sommes pas nihilistes, parce que nous avons certaines valeurs : la liberté, la justice ${ }^{7}$, ou lorsqu'il disait à la libération que «Paris se libérait non pour le pouvoir, mais pour la justice». Est-ce à dire qu'il faille passer par les valeurs comme positivité, pour échapper au néant d'une liberté-libération devenue par la force des choses négative? Le chrétien n'acceptera pas, au nom de ses valeurs la position du héros de Sartre sur l'avortement. Mais on peut, aussi défendre bien d'autres valeurs qui font l'essentiel de la vie et de l'existence.

34 N'y a t-il pas une positivité de la liberté ? Je me rappelle que j'étais tellement assailli par ce problème, que lorsque j'allais pour la première fois en 1973, questionner pour le bac, ce devait être au lycée Balzac, à une époque où il n'y avait pas de texte: j' interrogeais sur la liberté comme positive, lui promettant de lui mettre une bonne note, s'il réussissait à m'en faire ressortir les aspects positifs, il m'a parlé de la liberté dans l'action, ce que je savais, mais aussi de Bergson, de la liberté de la personne . Je retrouvais des aspects plus positifs à la liberté, celle de l'homme qui agit, de cette «liberté qui est la condition première de l'action », de cette liberté que j'espérais où l'homme arrive à se réaliser, ainsi que l'artiste par sa création, de cette liberté qui émane de la personne humaine toute entière.

35 Le problème de la liberté ainsi posé demeure celui de sa réalisation concrète : nous venons d'entrevoir tout un domaine d'extension, celui de la création artistique, que nous avions déjà posé dans une certaine mesure à travers la révolte des dissidents , pour une littérature libre, ou encore à travers Camus le cas d'une littérature engagée qui suppose en arrière-fond l'existence de la liberté pour dire et dénoncer les conditions de l'injustice, en outre que le fait même de la création est en soi un acte de liberté, ainsi qu'au XVIII è siècle qui se proclamait siècle des philosophes où la littérature " descend des cabinets de travail pour aller dans la rue sur les places publiques et dans les marchés $»^{8}$, devenant cette intense fabrique de la liberté. 
36 Mais il y en a d'autres, celles de toutes les autres libertés, telles qu'elles ont été posées dans les réponses à la question : celle des libertés concrètes : libertés à ne peut-être plus poser avec un L majuscule, d'une vision idéale mais au pluriel On a, en effet, besoin de préciser: de quoi parle-t-on, quand on parle de liberté, puisqu'on vu ce risque de tenir un discours qui ne serait que des mots, englué dans un idéalisme, sans débouché dans la vie concrète. Et les réponses précisent souvent : la liberté de penser, c'est dire d'avoir les opinions que l'on veut, ce qui n'est pas si évident dans un monde tel que l'indiquait Marcuse où l'opinion est façonnée par les médias, ou manipulée, ainsi que le notait le marxiste hongrois G. Lukacs: nous sommes à l'époque des manipulations ${ }^{9}$. Le dire marcusien est encore juste : il n'est pas si facile que cela d'avoir une opinion libre, quand la pensée unique veille, dans cette uniformisation des types de discours.

37 Liberté de la presse qui a été une liberté créée par les progrès de la civilisation. Il semblerait ainsi qu'à mesure de son développement celle-ci crée des libertés : liberté de voyager favorisée par les compagnies aériennes. On pourrait voir le progrès comme un épanouissement de chacune de ces libertés rendues possibles. Nous serions plus libre qu'à l'époque de Rousseau : ce n'est pas si sûr, car la liberté n'est plus celle d'un homme solitaire qui vivrait dans une île, ou dans son coin de terre où le paysan du XVIII è siècle pouvait après tout jouir d'une certaine autonomie, mais celle d'une société de dépendance et d'interdépendance.

38 Les libertés syndicales dans les entreprises, le droit de grève etc. ..., la liberté d'association, les libertés religieuses ou politiques dans leur définition dans le cadre d'une société démocratique, ces libertés qui ont été chèrement conquises, pour lesquelles, en effet, les moindres libertés ont été arrachées par le combat ou les luttes des hommes qui y ont contribué.

Conclusion

39 La liberté ainsi est à faire, et reste à faire, là où notre formule être libre c'est se libérer prend cette fois un sens devenu plus positif. La liberté est une conquête de l'homme et de l'histoire, de l'homme à travers une «histoire, qui n'a été que l'histoire du développement de la liberté parmi les hommes" ${ }^{10}$. On a vaincu l'esclavage et le servage, mais à chaque pas du progrès il faut veiller à la liberté qui reste toujours menacée et fragile, ainsi que le montrent les exemples les plus récents (lois informatiques et libertés et bien d'autres), car on risque toujours de transformer pour le progrès, celui-ci en un instrument et une machine, trouvera-t-on un robot qui pense à sa place, peut-on accepter le clonage humain? Il y va à chaque fois de la liberté qu'il faut savoir penser dans les termes de son temps. La liberté est celle de l'homme, car être homme c'est être libre, mais elle est à la mesure de son être toujours à travers les guerres, le développement des sociétés et des institutions politiques, à faire et à refaire, fragile et menacée. 


\section{NOTES}

1. G. W. Leibniz, Essais de Théodicée, respectivement, § 86, § 278, IIIè partie, et $\$ 288$.

2. J. P. Sartre, L'Etre et le Néant, p. 524.

3. A. Vergez et D. Huisman, Cours de philosophie, réédition. 1990, p. 360.

4. Album Sartre, Pléiade, p. 73.

5. R. Aron, Histoire et dialectique de la violence, 1972, p. 76.

6. E. Kant, La religion dans les limites de la simple raison, IVè partie, 2 è section, en note, Pléiade, t. III, p. 226

7. A.Camus, Discours de Suède, 10 décembre, 1957, Pléiade, Essais, p. 1073.

8. J.P.Sartre, Qu'est-ce que la littérature ?, Folio, p. 116.

9. Entretiens avec G. Lukacs, Cahiers libres, Maspéro, 1960, p. 126

10. G. W. F.Hegel, Leçons sur la philosophie de l'histoire, 1967, traduction Gibelin, p . 28.

\section{RÉSUMÉS}

Après avoir situé nos concepts comme historico-politiques, nous dirons que malgré la sémantique des discours, la liberté se trouve dans une intense contradiction: liberté, égalité, fraternité, mais en même temps la guillotine; de même dans Marcuse, le monde libre et les bombes. Aussi distinguons-nous, la liberté comme concept, et comme réalité. Comme concept, la liberté est issue (a) de la théorie du péché originel où Adam est bon et libre, (b) de la théorie du libre arbitre où elle devient pouvoir de choix. Comme réalité, elle est issue pour nous de cette liberté-libération de 1789, des révolutions, puis de 1945 et des mouvements de libération. Mais cette liberté devenue ainsi négative ne peut-elle pas, être, à certains endroits, positive?

Our concepts being situated as" politic and historical ", we shall say that, notwithstanding the semantic of discourses, freedom is in contradiction: freedom, equality, brotherhood, but in the same time guillotine; so as in Marcuse, free world, and bombs. So we shall distinguish freedom as concept, and as reality. As concept freedom is issued (a) from the theory of original sin where God made Adam good, but free, (b) from the theory of "free-arbitre", where, while, he can do the goodness, man commits bad; freedom is power of choice. As reality, freedom is issued for us, from this liberation of 1789 and revolutions, of 1945 and liberation's movements. But this one which is so negative, is not also, somewhere positive?

\section{AUTEUR}

\section{JEAN-MICHEL CHARRUE}

Jean-Michel Charrue est professeur de philosophie au Lycée Saint-Gabriel de Bagneux (92), et à l'ISC de La Ville du Bois. Ses publications principales sont : Plotin lecteur de Platon, Paris, publication Paris-X Nanterre et Les Belles lettres, C. E. A. , sous le patronage de l'association G. Budé, 1978, puis Les Belles Lettres, 1987, et 1993. Illusion de 
la dialectique et dialectique de l'Illusion, Platon et Plotin, Les belles Lettres, 2003, Collection d'études anciennes, G. Budé. Ammonius et Plotin, revue philosophique de Louvain, $\mathrm{n}^{\circ} 102$, 1, 2004, p. 72-103; Traduction et commentaire du traité 20 , Sur la dialectique, dans Plotin, Traité 7-21, GF, 2003. Plotin et Parménide, dans Diotima, 32, 2004. 